\title{
Annotation
}

Journal of Medical Genetics, 1977, 14, 362-370

\section{Application of chromosome banding techniques to the study of primary chromosome structural changes}

\author{
JOHN R. K. SAVAGE \\ From the MRC Radiobiology Unit, Harwell, Didcot, Oxon
}

SUMMARY Some comments are provided for the benefit of new workers on the use of chromosome banding techniques for the recognition, classification, scoring, and break point location of primary 을 chromosomal structural changes.

\section{Introduction}

Since the publication of the annotation on the classification and relations of induced chromosomal structural changes (Savage, 1976), I have had frequent requests for comment on the application of chromosome banding techniques to the study of induced aberrations. These requests have prompted me to provide a few simple notes which I hope will be of benefit to new workers.

The comments below concern primary aberrations, but many of the principles will apply to derived types encountered in clinical work. There are, however, a number of very important differences between these two categories with respect to the application of banding.

Firstly, many of the primary types, in particular the asymmetrical forms, do not require banding for detection, and in certain cases (e.g. interstitial deletions) banding may be a positive disadvantage, particularly in quantitative work.

Secondly, in the clinical situation, one has several chances to observe the same set of changes, so that variation between the banding quality of different cells can be overcome (or even used) when making the final decision. Moreover, there is the possibility of using several different techniques $(G, R, Q, C, T$, etc) on replicate samples of the same structural change, thus facilitating accurate identification. In sharp contrast, a particular set of primary changes is confined to one cell, in which the banding may be good, bad, or indifferent, and usually there is no second chance or alternative method possible to Received for publication 21 February 1977 confirm a particular interpretation (but see Verma $\stackrel{\mathbb{D}}{-}$ and Lubs, 1975; Buckton, 1976). This situation of course places a premium on technical excellence.

The decision to apply banding techniques to primary aberrations will be determined by the purpose of an experiment. For many purposes, for example radiation dose-response curves for biological dosimetry, little advantage accrues. There are three areas of interest where banding has been used:

(a) Detection and recognition of the nature of aberrations.

(b) Identification of the chromosomes involved.

(c) Location of the presumptive positions of the lesions involved in structural change (usually termed 'break points').

This annotation is concerned principally with (a) but brief comments will be made on (b) and (c). The various categories of aberration will be dealt with in the order given, and using the nomenclature of the previous annotation (Savage, 1976, referenced by I 9 followed by the paragraph number). Unless otherwise stated, remarks refer to G-banding produced by ASG or enzyme methods (Seabright, 1971; Sumner et al., 1971; Schnedl, 1973).

Bands that stain darkly (or fluoresce brightly) with a particular technique will be referred to as $d$-bands, while those that stain lightly or show dull fluorescence will be designated $l$-bands.

\section{Preliminary considerations}

The basic requirement for the detection of a chromosomal structural change using banding methods is a disruption in the normal sequential band pattern of a 
chromosome arm region. This disruption may take several forms, of which the commonest are: (1) an abrupt change to a foreign pattern, (2) an absence of expected bands, (3) presence of additional bands, and (4) reversal of a section of pattern.

Certain prerequisites are necessary for the fulfilment of this basic requirement.

\subsection{EXISTENCE OF CONSISTENT AND FIXED \\ PATTERN}

The consistency and universality of the G-band pattern for the human karyotype is amply documented, and some degree of fixity is implied in the construction of banding diagrams.

It must be remembered, however, that we are dealing with a dynamic situation, namely chromosome condensation, a process that occurs as the cell progresses towards metaphase, and that continues while cells are held at metaphase under the action of spindle-inhibiting drugs such as colcemid. Consequently, the number of bands, their pattern, and their clarity will be a function of the state of chromosome contraction. Many more G-bands can be seen in prometaphase chromosomes (Schnedl, 1971; Bigger and Savage, 1975) but the asynchrony between cells (and even between homologues within a cell), with respect to band fusions, precludes the idea of a fixed pattern. This asynchrony becomes less pronounced as condensation progresses, and is virtually absent by the time metaphase is reached, so that the pattern becomes more stable and, for practical purposes, fixed. With further contraction, band fusion continues until uniform staining density is reached.

The occurrence, therefore, of additional or missing bands, or of unexpected patterns, must always be weighed in the light of this dynamic situation.

\subsection{EXISTENCE OF GOOD SEQUENTIAL \\ DIFFERENTIATION BETWEEN BANDS}

Difference in size, staining intensity, longitudinal spacing, and numerical concentration of $d$-bands (and to a lesser extent $l$-bands) certainly exists, and forms the basis for the identification of individual chromosomes. Quality of differentiation varies between treatments (for example, ASG versus trypsin) and also between cells on the same slide.

In practice, the longitudinal differentiation in any one arm region is by no means as dramatic or obvious as the standard band diagrams imply, for these diagrams are a subjective 'average' based on the examination of many cells. The length of arm over which individual bands can be distinguished from each other differs considerably between different chromosome regions. Consequently for a particular aberration (e.g. a $2 d$-band inversion), the probability of detection will depend upon where, in the karyotype, it has occurred. In general, the smaller the length of run involved, the fewer the regions in which it will be seen. Obviously changes that have taken place wholly within a band seldom lead to pattern disruption (unless incomplete) and so remain undetected wherever they occur (see 5.3).

There will also be a difference between aberration types in this respect, for example, band deletions or additions are likely to be more obvious than inversions.

This variation in efficiency of detection between different regions of the karotype (regional detection variation) must always be borne in mind when considering the distribution of aberrations between chromosomes and in all cases of quantification.

\section{Detection and analysis of primary chromosomal aberrations}

\subsection{ACHROMATIC LESIONS (I, 3$)$.}

With ionizing radiations, the maximal yield of single achromatic lesions is found in the earliest cells to arrive in metaphase after irradiation. The observed frequency is drastically reduced in G-banded metaphases compared with conventionally stained preparations. This must mean that the majority of these lesions are located in $l$-band zones. It could also mean that achromatic lesions and $l$-bands are both manifestations of the same phenomenon, though this might be difficult to verify experimentally.

Nearly all chromatid gaps which occur in $d$-bands lead to chromosomal flexure, usually a 'dog-leg' bend (I, 6.3.1) and cannot therefore be distinguished from small chromatid intrachanges.

It seems that, at least for quantitative work with these aberrations, banding is contraindicated.

\subsection{CHROMOSOME-TYPE STRUCTURAL ABERRATIONS (I, 5) \\ 3.2.1 Asymmetrical interchange (I, 5.1.1)}

For detection of a dicentric, banding is unnecessary, but it can often reveal that an apparent simple dicentric is, in reality, complex, as for example two interchange events involving a common arm, or the conversion of an interarm-intrachange (centric-ring) to a dicentric by a second $\mathrm{A}$ or $\mathrm{S}$ interchange occurring within the loop region.

Such complex interchanges are not uncommon, and they are most easily recognized by the fact that the banding pattern of the acentric fragment does not correspond with that expected from the fusion of the two deleted distal regions of the chromosomes involved in the dicentric. It is therefore important to make a very careful analysis of both parts of the aberration. 
Dicentrics which have a large intercentromeric distance usually have very small fragments which, because they are predominantly terminal $l$-band material, will almost always be missed with $G$ (less frequently with R) banding. A proportion of fragment-less dicentrics is therefore to be expected in banded preparations (Seabright, 1973a).

\subsubsection{Symmetrical interchange (I, 5.1.2)}

Without banding the level of detection of reciprocal translocations is low. There are a number of factors which contribute to this. Obviously exchanges of equal lengths of terminal segments will be unnoticed because no change in chromosome arm lengths or arm ratios has taken place to alert the observer. However, even changes in length or arm-ratio are not sufficient in themselves for detection; the changes produced must result in anomalous morphology, otherwise the derivative chromosomes will simulate and be classified within standard Denver groups. Even when anomalous chromosomes are observed, accurate assignment of participants is seldom realised.

In contrast to the general experience with plant cells, banding studies have shown that most of the translocations induced by radiation in human cells do involve changes in arm-lengths and ratio (Seabright, 1973a; Buckton, 1976). Thus failure to recognise a translocation must arise from the misclassification of derivative chromosomes. Slight morphological differences are very easily hidden among the general length and arm-ratio variations which always occur within a cell.

Although banding removes much of the difficulty of recognition, the efficiency of detection is still not $100 \%$, primarily because two types are never detected: (a) those involving homologous bands of homologous chromosomes, and (b) those involving two terminal bands, for in neither case is the band pattern changed. For the remainder, efficiency of detection depends largely upon the lengths of the translocated segments, and to a limited extent upon which arms are involved.

As with dicentrics, banding frequently shows up complex translocations, for example those which contain within one arm segments intercalated from a third chromosome, or cases of multiple or cyclical exchange. Here again, the length of the segment involved is a key factor, and in general, the more complex the changes, the shorter the segment available for detailed study. Removal or intercalation of a single $d$-band ought, in theory, to be recognisable, but, in practice, unless it happens to be a very distinctive band (e.g. $7 \mathrm{q} 21$ or $14 \mathrm{q} 31$ ), it proves to be very difficult. Transference and intercalation of $l$-band material is even more difficult to pick up, because of the much greater range of size variation $\stackrel{\oplus}{+}$ normally found for these regions in different cells. $\Rightarrow$ As a consequence of these difficulties, most of these $\stackrel{\omega}{?}$ complexes are observed as a triad of non-reciprocalo translocations, 'one break involved in two different $\underline{\overline{\mathrm{D}}}$ events'. Such complex aberrations are more frequent $\frac{\bar{\sigma}}{\widetilde{\rho}}$ than one might expect. Seabright (1973a) recorded $5_{\varrho}^{\Phi}$ in a total of 28 primary translocations induced by $3.0 \mathrm{~Gy} X$-rays in human blood lymphocytes, and Buckton (1976) 8 in 66 after 2.0 Gy $X$-rays.

\subsubsection{Interarm intrachanges (I, 5.2.1).}

The A forms, centric rings, like dicentrics should be $e^{\text {. }}$ obvious without banding. However, the very great $\vec{P}$ discrepancies in published dicentric:centric-ring ${ }_{\omega}^{\circ}$ ratios in human cells (ranging from 3-17 for $X$-rays and up to $\sim 25$ for high LET radiations) suggest that ${ }_{0}$ difficulties in detection may be considerable.

These difficulties are likely to be aggravated rather $\vec{O}$ than relieved by banding. Very small rings, especially those from centric regions which do not stain darklyo with a particular technique, will be easily overlooked, $\stackrel{\Phi}{?}$ and much depends on the clarity of the acentric $\overrightarrow{0}$ portion to prevent other small centric rings being $\checkmark$ classified as interstitial deletions. As the mean aberration frequency increases, there is a preferentialo loss of larger rings ('diminution', Savage and Papworth, 1969) and their conversion to dicentrics by additional exchange events in the loop regions. $\frac{\mathcal{Q}}{\mathbb{Q}}$ Such derived dicentrics are usually obvious with banding since the band pattern of the fragment is not $\overrightarrow{\vec{O}}$ simply related to that of the dicentric.

The $\mathbf{S}$ form is a pericentric inversion and without banding only those which result in an obvious change in arm ratio will be seen. From our experience with fibroblasts from primary explants of humano skin and ASG banding, these constitute about $70 \% \frac{0}{3}$ of the total pericentric inversions observed. As with translocations, size and position are important ${ }_{3}$ factors for detection.

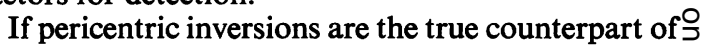
centric rings, then the size distribution of the latter $>$ leads to the inference that most of the small inversions (i.e. those involving only 2 or $3 d$-bands) are N missed, except in a few chromosomes where the band differences on either side of the centromere are 0 very obvious, for example 7,12 , and 16 . The number $\omega$ missed in this way is difficult to assess. If the probability of A:S exchange is assumed to be equal, ando the efficiency of detection of centric-rings is high, $\mathbb{D}$ then the ratio 'pericentric inversion/centric rings? would be a valid estimate within an experiment. Published values for this ratio are 0.91 (Seabright, $\frac{\vec{\Phi}}{\mathbb{D}}$ 1973a), 0.82 (Cooke et al., 1975), and 0.61 (Buckton, 1976). However, as noted above, the considerable $\underset{\propto}{\mathbb{\Phi}}$ 
discrepancies in the dicentric/centric-ring ratio suggest that the efficiency for scoring rings is variable, and that there will be a corresponding variation in the estimate for the efficiency of detection of pericentric inversions.

\subsubsection{Intra-arm intrachanges (I, 5.2.2)}

Interstitial deletions are easily recognized in conventionally stained preparations as paired dots or rings. Their modal diameter is very small for human cells, and the size distribution almost certainly goes below the limit of optical resolution, so that scoring efficiency is low and variable, a fact borne out by published frequencies.

Since many of the deletions are of sub-band size, there are a number of problems for their detection in banded preparations. Those containing a good proportion of $d$-band material have a reasonable probability of being observed provided that the banding method is not too disruptive of chromosome structure. In contrast, those entirely or predominantly l-band material will certainly be missed. A partial solution to this problem will be found in the $G \rightarrow R$ combination developed by Buckton (1976). For accurate quantitative work, however, banding must be regarded as a positive disadvantage.

An additional problem with interstitial deletions is that of location of the site of origin, since the majority do not remain associated with the parent chromosome, but lie free among the other chromosomes. For those containing a $d$-band, search must be made for missing bands, and a shortened chromosome, but unless it is a distinctive band, it is seldom possible to locate the position within the arm, even after diligent comparison with the undamaged homologue.

Another problem is that many small terminal deletions will be confused with interstitial ones and vice versa.

Chromosome type paracentric inversions, since they involve no change in chromosome morphology, or in the pairing affinity of sister chromatids, are never visible without banding in somatic cells. As might be expected from the size distribution of their A counterparts, the majority are also too small to be seen with banding. Even the bigger ones, unless they happen to occur in a very few favourable chromosome regions, lead to very little pattern disruption, and so are largely undetected. Paracentric inversions, therefore, are probably the most difficult aberrations to recognize and it is of interest to note that their recorded frequency is very low in clinical, as well as experimental, studies. In the human karyotype, very few chromosome regions possess sequential band differences of sufficient clarity to pick up any but the largest inversions, and a run of about $3 d$-bands is probably the minimum length required.
3.2.5 Breaks (I, 5.3)

As emphasised previously, the category of terminal deletions is very heterogeneous, and much can be done in a cell with high quality banding to assign fragments to the correct class of incompleteness.

The key is to make a very careful comparison between the pattern sequence of the fragment and the shortened parent (presumptive) chromosome.

Given a reasonable fragment size, the following are the most likely conditions found with primary aberrations. (N.B. these conditions are not applicable to second and subsequent division cells containing derived aberrations.)

(a) Fragment compound (usually containing two terminal regions); Suspect an incomplete dicentric or centric ring. (Compound fragment with a terminal and unrelated non-terminal region: incomplete complex interchange.)

(b) Fragment simple (material from one arm only) but with altered band sequence: sequence alteration is most frequently the inversion of a proximal segment $=$ incomplete paracentric inversion

(c) Fragment simple but the normal band sequence:

(i) Shortened arm has a normal band sequence: Fragment and shortened arm unrelated $=$ incomplete reciprocal translocation or pericentric inversion:

Fragment and shortened arm related $=$ true terminal deletion.

(ii) Shortened arm has abnormal band sequence:

distal inversion $=$ incomplete paracentric inversion distal material missing: incomplete interstitial deletion-look for minutes. additional distal unrelated segment $=$ incomplete complex change-look for origin of additional segment.

(d) Fragment simple, non terminal: incomplete acentric ring (i.e. large interstitial deletion).

An analysis such as that outlined above, presupposes not only a reasonable fragment size, but also very fine band differentiation. This is possible in practice only in less contracted prometaphase chromosomes treated for banding by the less vicious methods which do not disrupt chromosome structure. In practice, many primary deletions are rather too small for analysis following the usual prolonged period of colcemid metaphase accumulation.

It can be seen that the criterion given in I, 5.3, 'complete severance ... not associated with any obvious exchange process', can be applied much more rigorously when banding is present. However, even 
when pattern sequences appear to match perfectly, one can never be absolutely certain that the terminal deletion observed has not resulted from an incomplete intrachange too small to produce band sequence disruption.

\section{Chromatid-type aberrations (I, 6$)$}

Most kinds of chromatid type aberrations are recognisable without recourse to banding, so that the principal purpose in applying these techniques is for chromosome identification and 'break-point' location. Nevertheless there are occasions where banding can be of help in correct classification, and these are discussed below. Only techniques that produce minimal chromosome denaturation are suitable, i.e. where individual sister chromatids can be distinguished and traced throughout their length. This rules out most of the enzyme methods.

\subsection{INTERCHANGES (I, 6.1)}

There is a pronounced tendency for chromatid interchanges in mammalian cells to adopt a cruciform configuration (Wigglesworth and Savage, 1976), hence the term 'quadriradial'. This configuration, coupled with the very strong and precise pairing affinity of sister chromatids, enhances the ease with which pattern disruption is seen and break point positions determined (See Section 5.1). The chief advantage of banding lies in the resolving of complex interchanges, for it is now possible to trace the exact pathway of the various chromosome arms beyond the point of exchange.

\subsection{INTER-ARM INTRACHANGES (I, 6.2)}

At metaphase, banding does not help to distinguish between the two A-forms or between the two Sforms, but at early anaphase, careful sequence analysis can separate the S-forms, pericentric inversion and double duplication-deletion, and since the A-forms, dicentric and centric ring also become clear (I, 6.2.1), all four types can now be correctly classified.

\subsection{INTRA-ARM INTRACHANGES (I, 6.3)}

The majority of these are so small, that is the two lesions involved in intrachange are separated by such a short length of chromatid, that banding may be of no advantage in recognition, and in some cases may be a hindrance.

Taking the four simple types in turn:

(a) Isochromatid deletions (Revell type 4) are obvious without banding but the decision of incompleteness (NUp, NUd) is usually much more difficult to make in a banded prep- aration. Considerable difficulty can be experi enced in locating the breakpoints for the complete form (Savage et al., 1976, and se Section 5.1).

(b) The duplication-deletion (Revell type 1) is normally detected by an inequality in siste chromatid arm length, which shows as slight bump or 'dog-leg' bend. When a large segment is involved, pattern disruption is clear, but with smaller segments the tell-tale bending is lost as the result of the stretching and locat denaturation which invariably accompanies most banding procedures. Underestimatiors may, therefore, be expected.

(c) Chromatid minutes (Revell type 2). Much o $\vec{\beta}$ what was said about chromosome-type inter $\frac{\mathrm{C}}{\omega}$ stitial deletions (3.2.4) applies here also, an underscoring is expected. Larger ones will be detected, and in this case it is usually possible to differentiate between the two incomplete types $2 \mathrm{a}$ and $2 \mathrm{~b}$, and also to distinguish $2 \mathrm{aP}$ from 1a when the minute has been displaced응 (I, 6.3.2)

(d) The paracentric inversion (Revell type 3 ) almosto never seen in the absence of banding will be clear for larger segments in certain chromosome regions. The close proximity of an unchanged banded 'control' sister chromatid in the region of inversion enables very detailed band fon band comparison, so it is likely that the्D efficiency for detecting chromatid inversions is considerably greater than that for the corres? ponding chromosome types. In very favourable preparations, it is possible to distinguist between the two incomplete forms, a feat hitherto impossible.

\subsection{BREAKS (I, 6.4)}

Like the corresponding chromosome category chromatid terminal deletions are a heterogeneous mixture, though the components differ somewhat in the two categories.

Because of sister chromatid pairing affinityo incomplete interchanges and interarm intrachanges. do not contribute to chromatid 'breaks' (that is af metaphase of 1st post-induction division), so con $N$ tamination of the 'primary breaks' (if such exist, I을 4) comes principally from incomplete intrachanges ${ }_{5}^{\omega}$ both simple and insertion (I, 7.3).

Careful comparison of the patterns of both frage ment and parent is facilitated by the fact that the relevant pieces remain in proximity.

Sizes of intrachanges (4.3) preclude the use of banding to test Revell's theories, even assuming al intrachanges are simple. The five 'simple' contri $\frac{\text { P }}{\mathrm{D}}$ 
butors to the break categories (I, Fig. 5) will appear as follows:

1a: a tandem duplication present in the complete chromatid.

1b: a tandem duplication in either the centric or acentric portion of the incomplete chromatid.

2a: The origin of the acentric fragment is intercalary, the sister chromatid has a normal pattern, but may show bending opposite the site of deletion.

3a: a paracentric inversion adjacent to the break in the acentric fragment; sister chromatid normal.

3b: a paracentric inversion adjacent to the break in the centric portion; sister chromatid normal.

\subsection{ADDITIONAL CHROMATID ABERRATION} TYPES (I, 7)

Neither triradials nor isochromatid-isochromatid exchanges need banding for recognition. Occasionally additional $d$-bands are present in one of the chromatids at the exchange junction in the triradials indicating that at least one intrachange was involved in the formation of the aberration.

No assessment has yet been made of the frequency of insertion intrachanges in either irradiated or chemically treated human cells. Some of the more obvious types identifiable with conventional staining will be overlooked by banding, in particular where there is reduced staining of small deletions. Of the other types (many of which simulate simple intrachanges when seen at metaphase) very few will be distinguishable with banding, principally because insufficient bands are likely to separate the multiple lesions involved in the intrachange. Where, however, they do, very complicated pattern disruptions are to be expected.

\section{The location of aberration 'break points'}

At some stage in the production of an aberration, breakage and (usually) rejoining of the chromatid thread must occur and it is this event that can lead to the pattern disruption which we observe.

A detectable point of breakage is traditionally referred to as a 'break point' though in the light of the considerations discussed below, 'presumptive break point' would be more appropriate. Because of the precise longitudinal demarcation of the chromosome arm afforded by banding, these points can be mapped with reference to a given banding diagram. Nomenclature for desciptive mapping of derived changes is recommended in Paris Conference (1971), but as this method can be cumbersome for work with primary aberrations, simpler schemes have been suggested for experimental work where there is a lot of damage (Bigger et al., 1972; Savage et al., 1973a; Cooke et al., 1975; Seabright et al., 1975).

Mapping and associated chromosome participation studies present a number of pitfalls for the unwary and these are presented below as a series of cautions.

\subsection{CAUTION: THE 3-BAND UNCERTAINTY}

Given a pattern of light and dark bands (such as results from the various giemsa banding techniques), the human eye finds it much easier to distinguish gradations in size and staining intensity of the dark segments than of the light. Consequently, there is the tendency to concentrate attention on the disposition of $d$-bands, and to interpret pattern disruption solely in terms of $d$-band pattern. The use of the term 'interbands' for $l$-bands, often employed in conversation and publication (though ruled out by Paris Conference, 1971), underlines this natural preoccupation with $d$-bands. Conversely, with fluorescence techniques, the eye concentrates on the brightly fluorescing regions, with the same net result, since in the main they are equivalent to $\mathrm{G} d$-bands (Evans et al., 1971).

One immediate consequence of this is that the majority of presumptive break points for chromosome type changes (and therefore derived changes) appear to occur in, or are assigned to, the $l$-band where the discontinuity in $d$-band pattern occurs (Bigger et al., 1972; Holmberg and Jonasson, 1973; Jonasson and Holmberg, 1973; San Roman and Bobrow, 1973; Seabright, 1973a).

What is not generally recognized, however, is that there exists for nearly all chromosome-type aberrations an inherent 3-band uncertainty, for the same pattern disruption can be produced not only by an $l$-break/ $l$ break exchange where the pattern changes, but also by a $d$-break/d-break exchange in the bands on either side. The Figure illustrates this for a $6 ; 10$ reciprocal translocation using the Paris Conference (1971) band diagrams for these chromosomes. As mentioned above, $d$-band differences are seldom as distinct as the diagrams imply, and while it would be easy to cut the chromosomes in such a way as to make the distinction, this amounts to 'paper cytology' (Revell, 1974) and bears little relation to the actual object available for analysis.

It should be emphasised here that this ambiguity is not resolved by R-banding, for, assuming complete reciprocity for $G$ and $R$ bands within the region of interest, the sandwich of uncertainty just becomes $l-d-l$, and involves the same 3 bands (Fig. inset).

There will, of course, be some chromosome type aberrations where the particular bands or regions concerned allow only one solution, but these will be exceptions. Ambiguity is also eliminated in incom- 


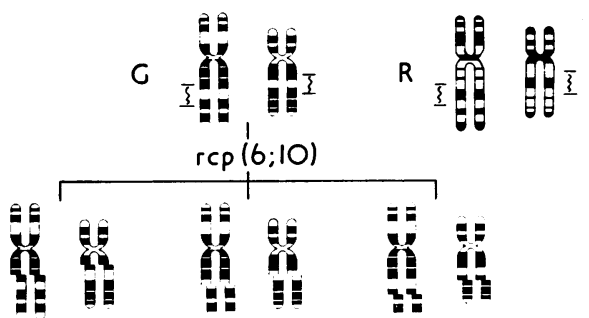

(q16;q21) (q21;q22) (q22;q23)

Symmetrical chromosome interchonge

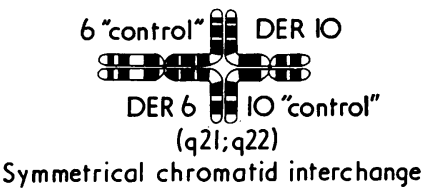

Fig. Diagrams of a reciprocal translocation to illustrate the 3-band uncertainty zone which invariably exists for locating break points in $G$-banded chromosome type aberrations. Inset shows that this ambiguity is not removed by $R$-banding. The lower figure shows how this uncertainty is overcome in chromatid type aberrations because the tenacious sister-chromatid pairing allows band for band comparison with an undamaged 'control' chromatid right through the region of exchange.

plete aberrations, but these are very rare for chromosome-types.

In contrast, little or no ambiguity exists for locating presumptive break points in the majority of chromatidtype changes (Savage et al., 1973b). Two factors combine to bring about this situation. Firstly, the very tenacious sister-chromatid affinity which retains close pairing, band for band, into metaphase, and secondly, the presence in most forms of an unchanged banded 'control' chromatid in close proximity to the 'break'. Thus the disposition of the bands can be traced and compared with the 'control' right through the break point region (Fig.). When this is done for radiation-induced interchanges in human lymphocytes, most of which adopt a cruciform ('quadriradial') configuration, the break points occur uniformly in $l$-bands (Savage et al., 1973b). As mentioned above, a similar finding has been frequently claimed for chromosome-type changes, but cannot, of course, be proved.

An additional bonus of help to location is that a reasonable proportion (10-25\%) of chromatid aberrations is incomplete $(I, 6)$.

A few chromatid aberrations do occur in $d$-bands after chemical treatment (Savage et al., 1976). Their rarity, however, is surprising when one considers the compound nature of all metaphase $d$-bands (Bahr and Larsen, 1974; Bigger and Savage, 1975). The low probability of $d$-band involvement is supported by the almost complete absence of $d$-band/l-band involve ment, which would be recognized by the creation of a new band at the site of exchange in one of the participating arms. It may be of course that when only part of a $d$-band region is transferred it is unstable, and tends to merge with adjacent $d$-bands.

Not all chromatid-type aberrations are unambefs guous with respect to locating break points. If particular, the isochromatid deletion in its complete form should be mentioned. In I, 6.3.1. it was pointed out that the loop origin of some of these may inferred from the eccentricity of the achromat胥 lesions which presumably occur at the site of ex. change. Such eccentricity will often pull bands round to occupy a terminal position at the zenith of the SUJ loop (see Savage et al., 1976, for diagrams) and cal lead to a false inference. The incomplete forms, NUD and NUd, are free from this problem.

\subsection{CAUTION: BAND REGIONS AND JUNCTIONS}

While it is convenient to think of the metaphase chromatids divided up into alternating $d$ - and $\frac{\vec{b}}{b}$ segments with sharp borders, as depicted on the majority of banding diagrams, we must not overloak the fact that in reality we are dealing with coile (Ohnuki, 1968) or possibly double coiled structures (Iino, 1971).

Typical G-band patterns are readily found amor⿳亠口冋 cells treated to display somatic chromosome cois, and the presence of intermediate patterns seems indicate some relations between condensation into gyres and bands. If this be so, it follows that a clegr $l-d$ band junction, at right-angles to the long-axis of the arm, would be more or less perpendicular to the gyre axis and may not necessarily demarcate a precise position on a coiled chromosome. If, as we believe, chromosome condensation is a dynamic process, तa given $l / d$ junction may represent different regions different cells.

In practical scoring terms this may not be of majör importance since we are working near the limits optical resolution, but recent improvements using $\mathbf{G} \rightarrow \mathbf{R}$ banding indicate that for some aberrations least, it is possible to locate a break point with suffis cient accuracy to distinguish between a position the middle of a band verses a $d / l$ band junctión (Buckton, 1976).

\subsection{CAUTION: Limits OF RESOLUTION}

There is now a sizeable body of evidence to implicate damage to the DNA and its repair with the formation of chromosomal structural changes under certaon circumstances. It is, however, all too easy to make the jump from molecular lesions to the changes that are 
observed in chromosomes with the light microscope, and overlook the fact that, even with banding, the resolution in molecular terms is extremely poor.

The human 2C DNA content is $\sim 5.8 \mathrm{pg}$ (Abrahamson et al., 1973), that is $2.9 \mathrm{pg}$ per chromatid set. The Harwell G-band norm (Bigger et al., 1972) has a total of $335 l$ and $d$ bands in a 9 chromatid set, so assuming a uniform distribution between all bands, we arrive at $8.66 \times 10^{-3} \mathrm{pg}$ per band. The total diploid length of DNA in $\beta$ configuration is approximately $1.93 \mathrm{~m}$ that is $2.88 \mathrm{~mm}$ per band (a scale model using cotton of $0.37 \mathrm{~mm}$ in diameter requires $\sim 354 \mathrm{~m}$ !).

Two things follow. Firstly, a large number of structural changes of considerable genetic significance could take place wholly within a band and, because they produce no pattern disruption, remain completely undetected. We are therefore only able to observe a proportion (probably a very small proportion) of the structural changes which actually occur.

Secondly, two independent, visually identical, aberrations involving break points in the same bands may be quite different in molecular terms and in genetical consequences. In passing it is worth noting that the probability of the same two $l$-bands being involved independently in another aberration (using equal weighting for Harwell $q$ norm) is $5 \times 10^{-11}$ so that the observation of such an occurrence can be taken as fairly strong evidence that the cells containing such have a clonal origin.

\subsection{CAUTION: BANDING AND QUANTITATIVE STUDIES}

Experiments using primary aberrations are usually undertaken with a view to quantitative study. It is important in such work to ensure that the cells which are scored are a truly representative and random sample from the population carrying induced structural changes. The warning regarding cell selection given $\mathrm{I}, 9 \mathrm{~b}$ is especially pertinent in the case of banded preparations. The technique employed to produce the various forms of giemsa banding are all to a greater or lesser extent, traumatic to chromosome structure, and the cell to cell variation, coupled with the need for complete karyotypes to analyse, leads to a much more rigorous cell selection. Consequently, the sample scored is frequently weighted in favour of less heavily damaged cells.

The milder methods of producing banding are, therefore, recommended in studies of primary aberrations, but it is difficult to see how this bias can be completely eliminated with techniques currently available.

I would like to acknowledge the valuable discussions and help afforded by the director Dr R. H. Mole and many other friends in the Unit during the preparation of the manuscript.

In particular I am indebted to Karin Buckton, Marina Seabright, Pat Cooke, Josette Derré, and my colleague Trevor Bigger, all of whom read the final draft, and provided some invaluable comments from their considerable experience in the field of banding and aberrations.

\section{References}

Abrahamson, S., Bender, M. A., Conger, A. D., and Wolff, S. (1973). Uniformity of radiation induced mutation rates among different species. Nature, New Biology, 245, 460-462.

Bahr, G. F., and Larsen, P. M. (1974). Structural 'bands' in human chromosomes. Advances in Cell and Molecular Bio$\log y, 3,191-212$.

Bigger, T. R. L., and Savage, J. R. K. (1975). Mapping Gbands on human prophase chromosomes. Cytogenetics ana Cell Genetics, 15, 112-121.

Bigger, T. R. L., Savage, J. R. K. and Watson, G. E. (1972). A scheme for characterising ASG banding and an illustration of its use in identifying complex chromosomal rearrangements in irradiated human skin. Chromosoma, 39, 297-309.

Buckton, K. E. (1976). Identification with $\mathrm{G}$ and $\mathrm{R}$ banding of the position of breakage points induced in human chromosomes by in vitro $\mathrm{X}$-irradiation. International Journal of Radiation Biology, 29, 475-488.

Cooke, P., Seabright, M., and Wheeler, M. (1975). The differential distribution of X-ray induced chromosome lesions in trypsin-banded preparations from human subjects. Humangenetik, 28, 221-231.

Evans, H. J., Buckton, K. E., and Sumner A. T. (1971). Cytological mapping of human chromosomes: results obtained with quinacrine fluorescence and the aceticsaline-Giemsa techniques. Chromosoma, 35, 310-325.

Holmberg, M., and Jonasson, J. (1973). Preferential location of X-ray induced chromosome breakage in the R-bands of human chromosomes. Hereditas, 74, 57-68.

Iino, A. (1971). Observations on human somatic chromosomes treated with hyaluronidase. Cytogenetics, 10, 286-294.

Jonasson, J., and Holmberg, M. (1973). Evidence for an inverse relationship between $\mathrm{X}$-ray induced chromatid and chromosome breakage in human chromosomes. Hereditas, 75, 259-266.

Ohnuki, Y. (1968). Structure of chromosomes. I. Morphological studies of the spiral structure of human somatic chromosomes. Chromosoma, 25, 402-428.

Paris Conference (1971). Standardization in human cytogenetics. Birth Defects: Original Article Series, 8, No. 7, 1972, The National Foundation-March of Dimes, New York.

Revell, S. H. (1974). The breakage-and-reunion theory and the exchange theory for chromosomal aberrations induced by ionizing radiations: a short history. Advances in Radiation Biology, 4, 367-416.

San Roman, C., and Bobrow, M. (1973). The sites of radiationinduced breakage in human lymphocyte chromosomes, determined by quinacrine fluorescence. Mutation Research, $18,325-331$.

Savage, J. R. K. (1976). Annotation: classification and relationships of induced chromosomal structural changes. Journal of Medical Genetics, 13, 103-122.

Savage, J. R. K., Bigger, T. R. L., and Watson, G. E. (1976). Location of quinacrine mustard-induced chromatid exchange points in relation to ASG bands in human chromosomes. In Chromosomes Today, Vol. 5, pp. 281-291. 
Ed. by P. L. Pearson and K. R. Lewis. John Wiley, New York.

Savage, J. R. K., and Papworth, D. G. (1969). Distortion hypothesis: an alternative to a limited number of sites for radiation-induced chromosome exchange. Journal of Theoretical Biology, 22, 493-514.

Savage, J. R. K., Watson, F. E., and Bigger, T. R. L. (1973a). A brief comparison of the Paris Conference (1971) and Harwell schemes for G-band numbering in human chromosomes. Chromosoma, 41, 123-127.

Savage, J. R. K., Watson, G. E., and Bigger, T. R. L. (1973b). The participation of human chromosome arms in radiation-induced chromatid exchange. In Chromosomes Today, Vol. 4, pp. 267-276. Ed. by J. Wahrman and K. R. Lewis. John Wiley, New York.

Schnedl, W. (1971). Analysis of the human karyotype using a reassociation technique. Chromosoma, 34, 448-454.

Schnedl, W. (1973). Giemsa banding techniques. In Chromosome Identification, pp. 34-37. Ed. by T. Caspersson and L. Zech. Academic Press, London and New York.

Seabright, M. (1971). A rapid banding technique for human chromosomes. Lancet, 2, 971-972.

Seabright, M. (1973a). High resolution studies on the pattern of induced exchanges in the human karyotype. Chromosoma 40, 333-346.

Seabright, M. (1973b). Non-involvement of the human $X$ chromosome in X-ray induced exchanges. Cytogenetics ands Cell Genetics, 12, 342-356.

Seabright, M., Cooke, P., and Wheeler, M. (1975). Variatione in Trypsin banding at different stages of contraction in human chromosomes and the definition, by measurement of the 'average' karyotype. Humangenetik, 29, 35-40.

Sumner, A. T., Evans, J. H., and Buckland, R. A. (1971) New technique for distinguishing human chromosomes Nature, New Biology, 232, 31-32.

Verma, R. S., and Lubs, J. A. (1975). A simple R banding technique. AmericanJournal of Human Genetics, 27, 110-117

Wigglesworth, D. J., and Savage, J. R. K. (1976). A comparison of the symmetry of radiation induced chromatid interchanges in animals and plants. In Current Chromo some Research, pp. 89-96. Ed. by $\mathrm{K}$. Jones and $\mathrm{P}$. Brandham. Elsevier/North Holland, Amsterdam.

Requests for reprints to Dr John R. K. Savage MRC Radiobiology Unit, Harwell, Didcot, Oxon OX11 ORD. 